\title{
A Trial of Parent Training for Mothers Being Released From Incarceration and Their Children
}

\author{
Ankie T. A. Menting and Bram Orobio de Castro \\ Developmental Psychology and Utrecht Centre for \\ Child and Adolescent Studies, Utrecht University \\ Leoniek D. N. V. Wijngaards-de Meij \\ Methodology \& Statistics of Social Science Research, Utrecht University \\ Walter Matthys \\ Department of Child and Adolescent Studies, Utrecht University \\ Department of Psychiatry, University Medical Center Utrecht
}

\begin{abstract}
Children of incarcerated mothers are considered at risk for disruptive behavior problems and later delinquency. Parenting may play a key role in this intergenerational transmission of delinquency. The present study aimed to evaluate the effectiveness of the Incredible Years parent training, enhanced with home visits, for (formerly) incarcerated mothers to prevent disruptive behavior problems in their 2- to 10-year-old children, by means of a nationwide randomized controlled trial. Mothers of 133 children ( $M$ age $=76.91$ months; $48.9 \%$ boys) were assigned to an intervention, consisting of group sessions and individual home visits, or a no-intervention control group. The intervention yielded significant effects on parenting and child behavior for maternal report. Marginally significant effects on child behavior were found for teacher report. The results show short-term effectiveness of parent training for the high-risk and hard-toreach population of (formerly) incarcerated mothers and their children.
\end{abstract}

The continuous cycle of delinquency passing from parents to children is one society would like to end. Children of delinquent mothers are considered one of the most at-risk populations for later delinquency (Lipsey \& Derzon, 1998; Murray, Farrington, \& Sekol, 2012). A recent meta-analysis (Murray et al., 2012) of the most rigorous studies to date showed children affected by parental incarceration had about a $10 \%$ increased risk of antisocial behavior compared to peers. Transmission of delinquency from delinquent mothers to their

The original research project is funded by the Dutch Ministry of Security and Justice, Directorate-General for Youth and Sanctions, Judicial Youth Policy Department. The funders facilitated recruitment but were not involved in recruitment, randomization, data collection, intervention, or preparation of the manuscript.

Correspondence should be addressed to Ankie T. A. Menting, Developmental Psychology, Utrecht University, PO Box 80140, 3508 TC Utrecht, The Netherlands. E-mail: A.T.A.Menting@uu.nl children appears to be at least as strong as transmission from fathers to their children (Bijleveld \& Wijkman, 2009). This intergenerational transmission is partly explainable through the accumulation of risk factors for later delinquency, and its precursor disruptive behavior problems, in these children's lives (Dallaire, 2007). It is this accumulation of risk factors across domains, rather than a single specific factor, that is important in determining adverse child outcomes (e.g., Sameroff \& Seifer, 1993).

Parenting is believed to be a mediating factor in the association between risk factors like parental delinquency, socioeconomic status, and maternal depression, and children's antisocial behavior and delinquency (Dodge, Coie, \& Lynam, 2006; Reid, Patterson, \& Snyder, 2002). Parenting has been demonstrated to partially mediate the relation between antisocial 
behavior in adolescent mothers and disruptive behavior problems in their children (Rhule, McMahon, \& Spieker, 2004). Furthermore, parenting behaviors were found to predict child behavior problems, although the association may be confounded by genetic factors (Moffitt, 2005), and parenting may be influenced by personal characteristics of both parents and the child. Poor monitoring and inconsistent discipline by mothers predict externalizing problems in their daughters and their children of both sexes respectively (Gryczkowski, Jordan, \& Mercer, 2010). Parenting dimensions of monitoring, psychological control, and negative aspects of support (neglect, hostility and rejection) predict delinquency (Hoeve et al., 2009). Parenting behaviors, in turn, also show evidence of continuity across generations (e.g., Conger, Neppl, Kim, \& Scaramella, 2003; Thornberry, Freeman-Gallant, Lizotte, Krohn, \& Smith, 2003). Thus, parenting may be important in maintaining the intergenerational cycle of crime.

However, parenting can also be used to break this intergenerational cycle. Interventions aimed at parenting behaviors have proven to be most effective in decreasing children's antisocial behavior (McCart, Priester, Davies, \& Azen, 2006), and in preventing children's antisocial behavior. Indeed, a number of prevention studies have successfully targeted parenting behaviors and children's disruptive behavior in children exposed to a variety of risk factors for antisocial behavior. Positive results were found regarding, for example, home visitation programs (e.g., Olds et al., 2002), Incredible Years (Webster-Stratton, Reid, \& Hammond, 2001), and the Oregon model of Parent Management Training (Bullard et al., 2010).

Although parenting programs have proven effective in high-risk populations, surprisingly few studies have targeted children of delinquent mothers, or even just families with delinquent family members. To our knowledge, only Brotman and colleagues's studies (Brotman et al., 2005; Brotman et al., 2003) examined the actual effectiveness of such a prevention program. The intervention included the Incredible Years parent training (Webster-Stratton, 2001). These studies, aimed at preschoolers in families with a history of antisocial behavior (mostly adjudicated older siblings), found intervention effects on parenting and child behavior. However, targeting delinquent mothers seems to be especially important, because of their significant role in parenting. Also it has been suggested that delinquent women experience more severe problems and risk factors than delinquent men (e.g., the gender paradox and higher thresholds for women [Loeber \& Keenan, 1994] and assortative mating [Simons, Stewart, Gordon, Conger, \& Elder, 2002]).

Certainly, intervention studies that directly address samples of incarcerated mothers or women who received an alternative sanction do exist (e.g., Cassidy et al.,
2010; Loper \& Tuerk, 2011). Also interventions have targeted related samples, such as families characterized by partner violence or child maltreatment (e.g., Hughes \& Gottlieb, 2004; Jouriles et al., 2009). However, in marked contrast to most interventions for nonincarcerated parents, studies regarding incarcerated parents typically do not assess effects on child behavior (besides parenting behavior). Typical outcomes for in-prison parenting classes include knowledge and attitude, mental well-being and parenting stress, and behavioral changes (such as recidivism rates) in parents (Loper \& Novero, 2010) but not in children. In general, these interventions consist of parenting classes, without guidance of a thoroughly specified theoretical model (J. M. Eddy, Kjellstrand, Martinez, \& Newton, 2010). These parenting classes typically offer plain instruction in generic communication and parenting techniques, with an overview of child development (J. M. Eddy et al., 2008).

Regarding parenting knowledge and attitudes, these studies revealed mixed results. With a pre-post design without comparison group, Browne (1989) found only negative changes in appropriate developmental expectations and alternatives to corporal punishment in a sample of 29 women who received an alternative sanction but had initially been incarcerated. Other authors found positive changes in appropriate developmental expectations (Harm \& Thompson, 1997; Palusci, Crum, Bliss, \& Bavolek, 2008; Thompson \& Harm, 2000), empathetic awareness of children's needs (Harm \& Thompson, 1997; Palusci et al., 2008), alternatives to corporal punishment (Harm \& Thompson, 1997; Palusci et al., 2008; Thompson \& Harm, 2000), and appropriate family roles and responsibilities (Harm \& Thompson, 1997; Thompson \& Harm, 2000), using similar pre-post designs. In a quasi-experimental design with 40 incarcerated mothers, Moore and Clement (1998) established no significant differences between the intervention group and comparison group regarding parenting and child-rearing attitudes and knowledge about behavioral management techniques. However, pre-post comparisons revealed increases in knowledge about positive child-management in the treatment group (20 mothers). No significant changes were noted for appropriate developmental expectations, empathetic awareness of children's needs, alternatives to corporal punishment, and appropriate family roles and responsibilities. Sandifer (2008) also used a quasi-experimental design but reported only pre-post comparisons. Similar to several pre-post studies, Sandifer revealed positive changes in appropriate developmental expectations, empathetic awareness of children's needs, alternatives to corporal punishment, and appropriate family roles and responsibilities in the treatment group (64 mothers). Furthermore, no significant change was found in the comparison group (26 mothers). 
In sum, these studies provide some evidence that interventions targeting female offenders can produce positive outcomes. However, it is unclear whether measured changes in metacognitive beliefs and knowledge represent true shifts in maladaptive attitudes, and whether changes in attitudes actually lead to changes in mothers' parenting behaviors, let alone changes in child behaviors (Loper \& Novero, 2010). Furthermore, most of these studies included small samples, and none of them included a randomized control group or examined child outcomes. In addition, most interventions provided broad psychoeducational support during a nonspecified period of incarceration.

Hence, more clarity about the effects of parent training for delinquent mothers is desirable. We have to know not only whether it is possible to increase knowledge, but, more important, whether it is possible to change actual parenting behaviors and child behaviors. Otherwise, the possibility to end the intergenerational cycle of crime by means of a parent training will remain unclear.

In endeavors to break this intergenerational cycle, early prevention seems obvious. Not only are the origins of trajectories of disruptive behavior to be found in early childhood (Broidy et al., 2003; Shaw, Lacourse, \& Nagin, 2005), but antisocial behavior is also difficult to treat once fully developed (e.g., Goldstein, Dawson, Smith, \& Grant, 2012). Moderate effects are found for interventions for antisocial children and adolescents, with parent training being the most suitable and effective for preschool and school-aged youth (McCart et al., 2006). Therefore, parent training aimed at preschool and school-aged children of delinquent mothers may be successful in breaking the intergenerational cycle of crime.

For parent training, issues regarding delivery of the parent training may be crucial. First, the exact timing of the intervention may be crucial for effectiveness. It seems logical to start interventions during imprisonment, when access to this hard-to-reach-population is easiest, and because of the possibility to work on problems specific to the mother (e.g., depression) before return to the family. However, interventions should preferably continue after release from incarceration. First and foremost, reentry is a difficult process for many women, so support seems to be warranted to prevent relapse. Second, the home situation is where mothers actually put their parenting behaviors in practice. Thus, most difficulties may become apparent for the mothers after reentry into society. Their return to the family is also a unique opportunity to practice parenting behaviors and adjust them if necessary. Hence, the period around release from prison seems an ideal moment for commencement of parenting interventions.

Furthermore, adaptation of the trainers' approach and training content to the population of incarcerated mothers may be essential in delivering an effective intervention. Concerning the trainers' approach, a collaborative approach seems to be most suitable for hard-to-reach, troubled families (Barlow, Kirkpatrick, Stewart-Brown, \& Davis, 2005). The intervention has to be flexible enough to be used within a diverse cultural population. Empowerment of parents' insights and acknowledgment of parents' unique strengths may be needed to build a nonblaming relationship, based on trust and open communication, with mothers who may be skeptical about outsiders who address sensitive topics (B. A. Eddy, Powell, Szubka, McCool, \& Kuntz, 2001) or reluctant to accept ideas taught in a "school-like" way. Addressing attitudinal and cultural barriers may be needed to reveal reasons for resistance and enhance engagement. Concerning training content, the training should exceed conventional psychoeducation and should incorporate practice of parenting skills. Moreover, the myriad of practical issues that these mothers confront when returning home may hinder the transference of information related to parenting skills. Immediate life stressors are often prioritized above long-term goals for children or relationships with children, and creative problem solving may be needed to make practicing parenting behaviors possible within hectic life schedules. Therefore, addressing the mother's own issues and contextual challenges, in addition to parenting issues, may be essential to improve her ability to parent her children (Boudin, 1998; J. M. Eddy et al., 2010).

The Incredible Years parent training (IYPT) seems a suitable intervention approach for this population because the manualized intervention with demonstrated effectiveness (Menting, De Castro, \& Matthys, in press) can be tailored according to each individual family's needs, each parent's cultural background experiences, education, knowledge, and values (Webster-Stratton, 2009). Furthermore, the collaborative and empowering IYPT approach fulfills aforementioned conditions regarding optimal communication to the population of incarcerated mothers. Therefore, in the present study, the IYPT was delivered to incarcerated and formerly incarcerated mothers. Besides IYPT group sessions, the intervention encompassed home visits, which followed the IYPT group sessions. These home visits were added to the group sessions, enabling addition individual work with mothers. This individual approach enabled more tailored coaching regarding parenting and, moreover, more attention for mothers' individual issues and contextual challenges.

The aim of this study was to evaluate the preventive effectiveness of the IYPT, enhanced with home visits, for disruptive behavior of 2- to 10-year-old children of mothers being released from incarceration, by means of a nationwide randomized controlled trial. We hypothesized that the intervention would have 


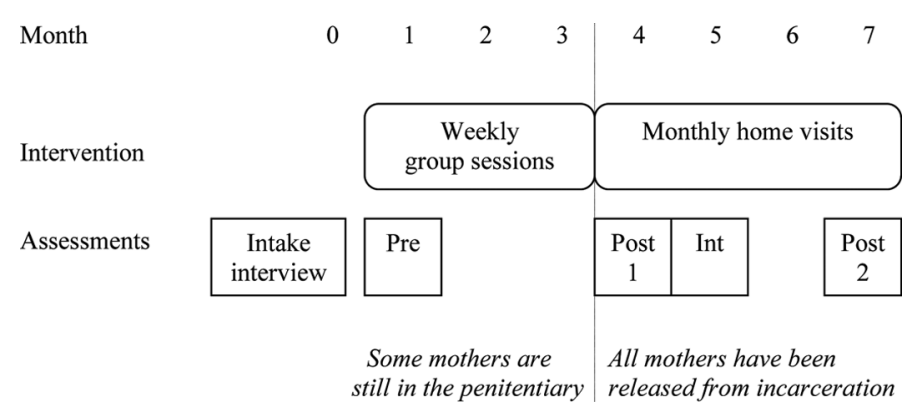

FIGURE 1 Intervention and measurement occasions. Note: Pre = preintervention; Post $1=$ postgroup sessions; Int =intermediate; Post $2=$ postintervention.

immediate effects on disruptive child behavior, according to mothers and according to teachers or childcare staff blind to intervention status, and on parenting behavior.

\section{METHOD}

\section{Design}

This study's design has two conditions (intervention group and control group), four measurement occasions within subjects, and two informants (mother and teacher/childcare staff). Individual mothers were randomly assigned to the intervention group or to the control group, in a 2:1 ratio with a simple randomization procedure (a throw of the dice by the second author, who was blind to participant information). ${ }^{1}$ To recruit sufficient numbers of participants, we planned to start group training half yearly over a period of 3 years. Therefore, participants were recruited in six recruitment periods.

However, some important changes to the methods had to be made after trial commencement. During two of the six recruitment periods for intervention groups there appeared to be too few potential participants to conduct the group training sessions properly. Therefore, for those two cohorts (concerning $24.7 \%$ of participants), randomization was temporarily suspended and all participants were assigned to the intervention condition. The lower number of potential participants in these two periods was not study or participant (approach, consent rate, etc.) related, but simply due to a smaller number of mothers ending their prison sentences in these periods. Furthermore, participants who were assigned to the intervention group but chose not to attend the intervention were invited to remain in

\footnotetext{
${ }^{1}$ A $2: 1$ allocation ratio was chosen to ensure sufficient group size within the group sessions. The IYPT requires at least six parents to optimize group discussions and to foster a sense of support (Webster-Stratton, 2001).
}

the study. Assessment in this "opt-out group" was relevant to us, because this enabled us to use real data instead of imputed data for these participants in intention-to-treat analyses.

Following an intake interview, four measurement occasions were included in the study (see Figure 1). Assessments took place at the start of the intervention (preintervention), after completion of the group sessions (4th month of the intervention; postgroup sessions), and after completion of the intervention (postintervention). In addition, an intermediate assessment was taken in the 5th month of the intervention (intermediate). This intermediate assessment was added to increase statistical power and chances to stay in touch with mothers after their incarceration.

If mothers met criteria (see participants) regarding more than one child, mothers were invited to provide information about three children, maximum. If children went to school or childcare and mothers consented approach, the children's teachers and childcare staff were asked to complete questionnaires. Teachers and childcare staff were blind to allocation status, and received a letter stating that the research was aimed at mothers in a difficult situation.

\section{Participants}

Incarcerated and recently released mothers were recruited through nationwide screening within all penitentiary institutions or via organizations whose clientele partly consists of formerly incarcerated women. Within the penitentiaries, monthly nationwide screenings, based on the total population of female inmates, were undertaken to trace all possible participants in the Netherlands between July 2007 and April 2010. Mothers had to meet three inclusion criteria. First, mothers had to be either incarcerated and expecting release within 3 months or formerly incarcerated and recently released (i.e., not exceeding 6 months). Mothers' release had to be within 3 months to enable home visits to be conducted as part of the second part of the intervention; all mothers had to 
be released from incarceration before postgroup sessions. Second, after incarceration, they had to be (expected to become once again) caregivers (at least weekly contact as a coparent) for their children aged between 2 and 10 years. Third, during the period of the IYPT group sessions, they had to be able to see their children during at least two weekends per month. Because most participants $(95.6 \%)$ were the biological mother of at least one of the participating children ( $M$ age $=76.91$ months, $S D=33.07 ; 48.9 \%$ boys), the term "mothers" is used throughout this article.

Mothers were convicted to a sentence of, on average, 11.1 months (range $=0.3-57$ months). The majority of mothers $(57.5 \%)$ were convicted of drug-related offenses, and for most mothers $(68.9 \%)$ this conviction led to their first incarceration. During incarceration, mothers called their children on average 4.8 times per week $(S D=5.3)$. However, $9.6 \%$ of mothers did not have any telephone contact with their children. On average, children visited their mothers monthly $(S D=1.3)$. However, $33.3 \%$ of mothers were not visited by their children during incarceration. Most mothers (55.8\%) were able to visit their children during their weekend leave at some point in their incarceration. During the intake interview, $58 \%$ of all mothers were already released from the penitentiary and were therefore able to see their children more frequently.

Most mothers (73.6\%) were low educated: $3.8 \%$ did not complete primary education, $48.1 \%$ only completed primary education, and $21.7 \%$ only completed lower secondary education. A minority $(23.6 \%)$ was native Dutch. $^{2}$ The other mothers originated from the Caribbean (35.8\%), South America (32.1\%), other European countries (3.8\%), Africa (1.9\%), Asia (1.9\%), or North America $(0.9 \%)$.

During the intake interview, the following problems, past and present, were reported: $34 \%$ maltreated, $16 \%$ sexually abused, $11.1 \%$ raped, $19 \%$ custodial placement of a child, and $22.3 \%$ incarceration of a parent. During baseline assessment, mothers reported adverse socioeconomic circumstances: $38.5 \%$ reported having no house (and having to live with friends or family after incarceration), $87.5 \%$ reported having debts, $27.1 \%$ reported having to live on social security benefits, and $46.9 \%$ reported having to live without social security or (partner's) income. Furthermore, mothers reported high levels of maternal distress, including depression (Menting, De Castro, \& Matthys, 2012).

\footnotetext{
${ }^{2}$ We used the customary definition of foreigner in the Netherlands (Keij, 2000), which says that a person is considered a foreigner if at least one parent was born abroad. That is, all mothers who were not considered foreigners were considered native Dutch.
}

\section{Procedure}

Participation in the trial was voluntary for all participants. All participants were assured of confidentiality and that the data would be processed anonymously. This assurance included a promise that no information traceable to individual participants would be shared with the Ministry of Security \& Justice or the penitentiary institutions. Prior to participation, mothers signed an informed consent form. After consent, randomization took place and an intake interview $(1.5 \mathrm{hr})$ was conducted. Remaining measurements were taken during three face-to-face assessments $(1 \mathrm{hr}+0.5 \mathrm{hr}$ for each extra child) and one intermediate assessment ( $20 \mathrm{~min}+15 \mathrm{~min}$ for each extra child); see Figure 1. All questionnaires were administered individually and mostly in an interview format. For the face-to-face assessments, mothers were visited at home or in the penitentiary by teams of two project members. If mothers were not present at the agreed time and place, several attempts were undertaken to conduct the assessment as soon as possible. Mothers received a monetary compensation for the time spent completing questionnaires at the four assessments. For the intermediate assessment, mothers received a monetary compensation of $€ 5$ (i.e., \$6.50) per child. For face-to-face assessments, monetary compensations rose per assessment, from $€ 20$ (i.e., \$26) per child at preintervention to $€ 50$ (i.e., \$65) per child at postintervention. Questionnaires were sent to teachers and childcare staff after face-to-face assessments with mothers. The study was approved by the Ethics Committee of the Utrecht University Faculty of Social Sciences.

\section{Intervention}

The intervention "Better Start" included 12 weekly 2-hr group sessions for mothers and four 1.5 -hr home visits per mother. Six groups of mothers received the group sessions in different towns and cities across the Netherlands. One group received the sessions within a penitentiary, whereas the other groups received the sessions in community centers. During group sessions, the BASIC IYPT (Webster-Stratton, 2001) was delivered. The BASIC IYPT is a manualized group parent training in which parents of young children view videotapes depicting parent models interacting with their children in various situations. In collaboration with two group leaders, mothers discussed these video vignettes and put learned techniques into practice in role-plays. The topics play skills, praise and rewards, limit setting, and handling misbehavior were discussed. Mothers were taught to use child-directed play skills, less critical and harsh discipline, and more positive and consistent strategies. In addition, mothers read the Dutch translation of 
the book The Incredible Years: A Trouble-Shooting Guide for Parents of Children Ages 3-8 Years (Webster-Stratton, 1992), and home assignments were used to encourage mothers to practice parenting skills at home. Due to some mothers' reading difficulties, handouts with chapter summaries were provided where chapter content was shortly reproduced in simple language, ending with a one-page summary.

Home visits were added to the group sessions to practice parenting skills, to support mothers to use adequate parenting skills in difficult individual circumstances, and to provide individual practical consultation. Mothers received home visits in the 4 to 6 months after completion of the group sessions. In addition to individual difficulties, home visits covered two subjects within the ADVANCE IYPT (Webster-Stratton, 2002) which elaborates on the BASIC IYPT. During the second home visit, communication with adults and children was addressed, and problem solving with adults and children was addressed during the third home visit. During home visits, but also during group sessions, mothers received practical consultation if desirable (e.g., regarding debts and social security).

The intervention was delivered by four team members with backgrounds in child psychology or within women's penitentiaries who had received a 3-day training workshop. Treatment fidelity was ensured by at least one IYPT certified group leader delivering all group sessions. Two team members became certified group leaders prior to delivering the groups investigated in this study. In addition, one team member became a certified group leader after delivering several groups investigated in this study, as a cotrainer with a certified trainer. Furthermore, the group leaders received supervision from accredited IYPT trainers, and group sessions were videotaped then reviewed during weekly meetings to ensure treatment fidelity. In addition, the manual of the BASIC IYPT was used, and both parental evaluations and checklists for group leaders were completed after the group sessions. Treatment fidelity, as measured by these checklists, was satisfactory: Overall, group leaders reported that they accomplished $98.1 \%(S D=3.36$; range $=84.2-100.0)$ of the activities.

In the control condition, the same assessments were administered as in the intervention group. Both families from the control condition and families from the intervention condition were allowed to receive usual services. In addition, trainers and researchers offered their help in finding adequate services when needed, in both conditions.

\section{Measures}

Basic Demographics and Family Functioning. General background information regarding mothers, children, and circumstances within these families were assessed with a basic demographics and family functioning form. The amount of contact between mothers and children was assessed repeatedly to enduringly check criteria fulfillment.

Eyberg Child Behavior Inventory (ECBI). The ECBI (Eyberg \& Pincus, 1999) is a questionnaire designed to measure parental reports of children's problem behaviors in children aged 2 to 16 years. The ECBI consists of 36 items rated on two scales: an intensity scale measuring the intensity or frequency of the problem behavior on a 7-point scale (never to always), and a problem scale, measuring the extent to which this behavior is a problem for the parents (yes or no).

In the present study, the ECBI was completed during each assessment. Internal consistencies were adequate for both scales, with Cronbach's alphas ranging from .89 to .93 across assessments.

Teacher's Report Form (TRF) and CaregiverTeacher Report Form (C-TRF). Children's teachers or childcare staff were asked to complete the Teacher Report Form (Achenbach \& Rescorla, 2001) or Caregiver-Teacher Report Form (Achenbach \& Rescorla, 2000), which is the preschool version of the TRF. The TRF consists of 113 items assessing behavior problems in children aged 6 to 18 years. The C-TRF consists of 100 items assessing behavior problems in children aged 1.5 to 5 years. For each item, childcare staff and teachers circled the answer (never, sometimes, or always) that fitted the behavior of the child in the preceding 2 months. To compare TRF and C-TRF scores we used $T$ scores in our analyses. For convenience, we use (C)TRF to refer to $T$ scores on both TRF and C-TRF.

In the present study, the aggressive behavior scale was used to measure behavior problems according to teachers or childcare staff. Internal consistencies for C-TRF and TRF were adequate, with Cronbach's alphas ranging from .86 to .97 across assessments.

Alabama Parenting Questionnaire (APQ). The APQ (Shelton, Frick, \& Wootton, 1996) is a self-report questionnaire designed to measure the most important aspects of parenting behaviors related to disruptive behavior problems in children. The APQ's 42 items are divided into the following scales: involvement (10 items), positive parenting (six items), poor monitoring/supervision (10 items), inconsistent discipline (six items), corporal punishment (three items), and other discipline practices (seven items). However, in the current study, two items were deleted: "You attend PTA meetings, parent/teacher conferences, or other meetings at your 
child's school" (involvement) and "Your child fails to leave a note or to let you know where he/she is going" (poor monitoring). This was because some participants were incarcerated at preintervention; thus, the unfeasibility of these items was deemed too confronting. Participants responded on a 5-point frequency scale (never to always).

In the present study, the APQ was assessed during all face-to-face assessments. Internal consistencies varied across assessments, with Cronbach's alphas ranging from .48 to .80 .

\section{Data Analysis}

To account for the multilevel structure of the data (assessments within children within mothers), multilevel analyses were performed in HLM 6.08 (Raudenbush, Bryk, \& Congdon, 2004) to examine change over time. Specifically, three-level models were used to examine intervention effects on preintervention to postintervention changes in child behavior and parenting, except for positive parenting and corporal punishment. ${ }^{3}$ In the three-level models, assessments (Level 1) were nested within children (Level 2) and children were nested within families (Level 3). For positive parenting and corporal punishment, two-level models were used because of nonsignificant variance at the child level. In these models, assessments (Level 1) were nested in families (Level 2). Because four scales (ECBI-problem scale, (C)TRFaggressive behavior, APQ-poor monitoring, and APQ-corporal punishment) were not normally distributed, results were reported based on robust standard errors for these scales. The intervention variable (intervention [1] vs. control [0]) was entered at the family level. Assessment waves were coded 0, 3, 4, and 6 reflecting the time schedule of assessments. In per-protocol analyses, children whose mothers participated in at least one session of the intervention were included in the intervention group. Thus, the opt-out group was excluded in per-protocol analyses. However, in intention-to-treat analyses, children from mothers who were invited to participate but never attended any sessions were added to the intervention group. Therefore, intention-to-treat analyses reflect actual randomization. Preintervention to postintervention effect size estimates $(d)$ were calculated for significant Group $\times$ Time interactions.

\footnotetext{
${ }^{3}$ Four-level MLwiN models were run as part of preliminary analyses to examine the nesting of families within groups (i.e., families were part of one of six intervention groups, five opt-out groups or four control groups). Because variance at the group level did not exist in 11 of 16 analyses, and did not exceed $5 \%$ of total variance in three other analyses, group level was not included in the final models.
}

\section{RESULTS}

\section{Participants}

Nationwide screening resulted in 183 mothers who seemed eligible for the study (see Figure 2). Of those 183 mothers, 129 actually met criteria and were asked to consent, and $113(87.6 \%)$ of them actually chose to participate.

Contact with seven mothers was lost immediately after informed consent. Furthermore, regarding eight mothers with nine children, further assessments were not possible due to loss of contact despite numerous attempts. Data of seven mothers were excluded from analyses because these mothers did not fulfill inclusion criteria in retrospect. That is, data of six mothers were excluded because they were not considered to be caregivers (i.e., there was no weekly contact at postgroup and postintervention), whereas data of one mother was excluded because her child turned out to be older than 10 years old at the start of the intervention. Therefore, data regarding 133 children of 91 mothers were available for the intention-to-treat analyses, whereas per-protocol analyses involved 102 children of 72 mothers. For report by teachers and childcare staff, data regarding 106 children were available for the intention-to-treat analyses, whereas per-protocol analyses involved 80 children. In many cases teachers or childcare staff could not be approached: Mothers dropped out or temporary loss of contact with mothers $(21.0 \%)$, young children did not have a teacher or daycare provider (11.7\%), teachers were absent during summer holidays $(9.3 \%)$, or permission to approach teachers or childcare staff was not obtained $(7.7 \%)$. No baseline differences between children with and without teacher data were found, except for children's age, that is, children without teacher data were younger than children with teacher data. In addition, no baseline differences between children from the intervention group and the control group were found regarding parenting and child outcomes.

\section{Attendance}

The mean attendance during 12 group sessions was 7.7 sessions $(S D=3.1)$, with six mothers $(12.2 \%)$ attending one to three sessions and 19 mothers $(38.8 \%)$ attending 10 to 12 sessions. Mothers in the intervention group received on average $3.2(S D=1.4)$ of four home visits, five mothers $(10.2 \%)$ received no home visits, and 34 mothers $(69.4 \%)$ received four home visits. Mothers were included in analyses irrespective of the amount of intervention received (see data analysis).

\section{Main Analyses}

Table 1 provides descriptive statistics for intervention, opt-out and control groups at preintervention, postgroup 


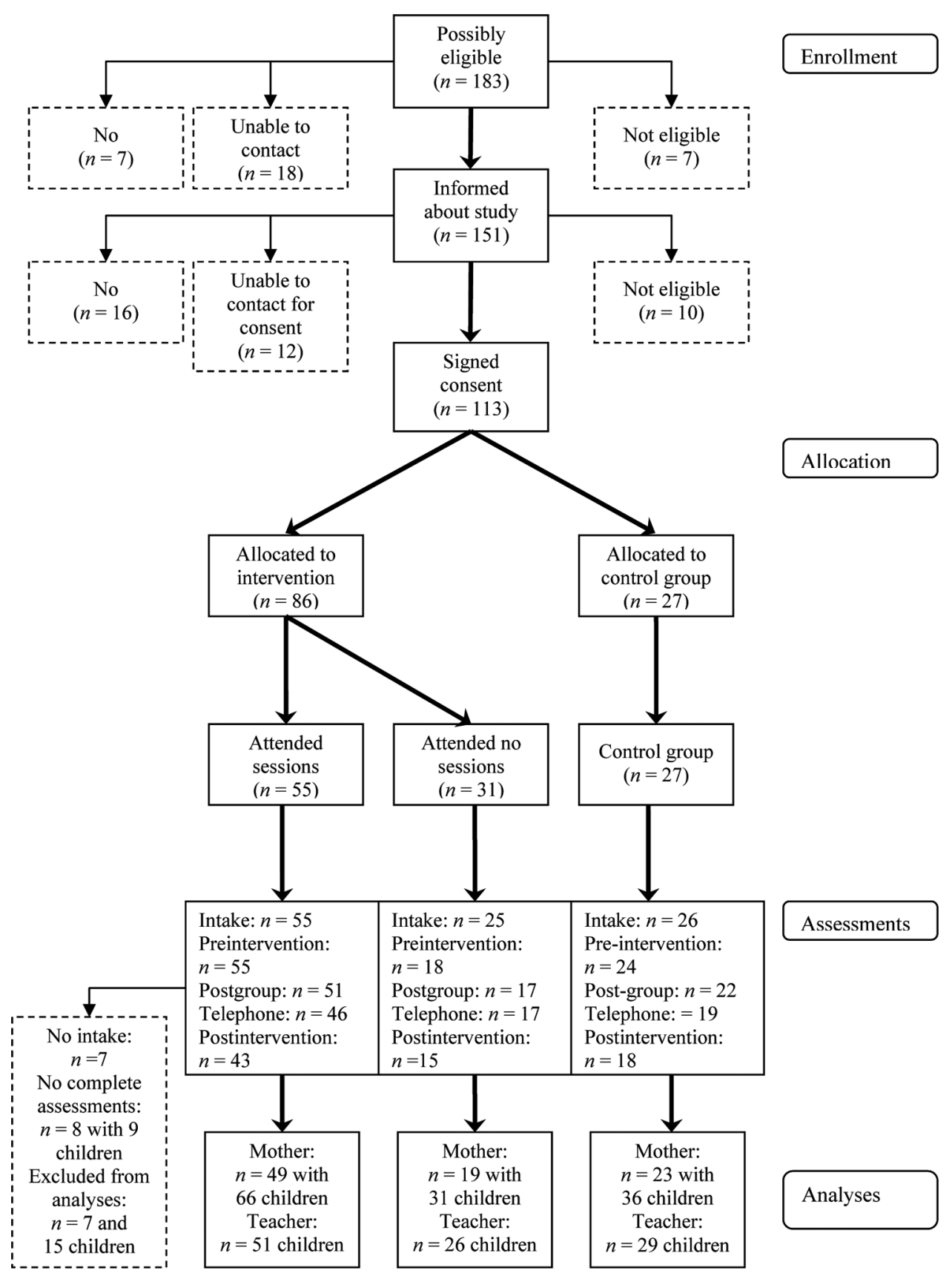

FIGURE 2 Participant flow. Note: The $n$ s refer to the mothers, except for the analyses as displayed at the bottom of the figure. Mother $=$ analyses regarding questionnaires filled out by mothers (Eyberg Child Behavior Inventory and Alabama Parenting Questionnaire); Teacher = analyses regarding questionnaires filled out by teachers and childcare staff (Caregiver-Teacher Report Form or Teacher Report Form).

sessions, intermediate, and postintervention assessment. Results of analyses are presented separately for disruptive child behavior and parenting behaviors. Intention-to-treat analyses are presented before perprotocol analyses.

Because we were especially interested in the effectiveness of the intervention, our main interest in analyses was whether the slope across measurement occasions differed between the intervention and the control group.
Such significant Group $\times$ Time interaction within models indicates that group status explains variance between families. Hereby, positive significant regression coefficients indicate an increase in the dependent variable in the intervention group when compared to the control group, whereas negative regression coefficients indicate a decrease for the intervention group. However, it is relevant to test for Group $\times$ Time interactions only if variance across time differs between families, that is, if 
TABLE 1

Descriptive Statistics for Child Behavior and Parenting Behaviors

\begin{tabular}{|c|c|c|c|c|c|c|c|c|c|c|c|c|}
\hline \multirow[b]{2}{*}{ Variable } & \multicolumn{3}{|c|}{ Preintervention } & \multicolumn{3}{|c|}{ Postgroup Sessions } & \multicolumn{3}{|c|}{ Intermediate } & \multicolumn{3}{|c|}{ Postintervention } \\
\hline & $M$ & $S D$ & $n$ & $M$ & $S D$ & $n$ & $M$ & $S D$ & $n$ & $M$ & $S D$ & $N$ \\
\hline \multicolumn{13}{|l|}{ Intervention Group } \\
\hline \multicolumn{13}{|l|}{ Disruptive Child Behavior } \\
\hline ECBI-Intensity & 111.84 & 30.47 & 65 & 104.24 & 26.13 & 62 & 99.24 & 27.78 & 56 & 94.23 & 24.27 & 53 \\
\hline ECBI-Problem & 13.61 & 8.74 & 65 & 12.20 & 7.17 & 61 & 11.18 & 7.61 & 56 & 10.37 & 7.86 & 53 \\
\hline (C)TRF-Aggressive Behavior & 58.63 & 7.96 & 38 & 60.36 & 11.41 & 22 & & & & 58.13 & 8.86 & 39 \\
\hline \multicolumn{13}{|l|}{ Parenting Behaviors } \\
\hline APQ-Involvement & 30.13 & 5.33 & 66 & 30.58 & 4.91 & 62 & & & & 30.94 & 4.55 & 52 \\
\hline APQ-Positive Parenting & 25.18 & 3.08 & 66 & 24.85 & 3.07 & 62 & & & & 24.87 & 3.50 & 53 \\
\hline APQ-Poor Monitoring & 12.37 & 3.55 & 65 & 12.25 & 3.66 & 62 & & & & 11.66 & 2.56 & 52 \\
\hline APQ-Inconsistent Discipline & 14.91 & 3.70 & 66 & 13.77 & 3.44 & 62 & & & & 12.43 & 3.37 & 53 \\
\hline APQ-Corporal Punishment & 4.94 & 1.84 & 66 & 4.69 & 1.65 & 62 & & & & 4.64 & 1.93 & 53 \\
\hline \multicolumn{13}{|l|}{ Opt-Out Group } \\
\hline \multicolumn{13}{|l|}{ Disruptive Child Behavior } \\
\hline ECBI-Intensity & 91.78 & 24.95 & 29 & 100.09 & 27.30 & 28 & 91.77 & 24.53 & 28 & 92.42 & 27.59 & 25 \\
\hline ECBI-Problem & 4.39 & 3.79 & 29 & 5.57 & 5.98 & 27 & 5.62 & 5.48 & 28 & 5.21 & 6.33 & 25 \\
\hline (C)TRF-Aggressive Behavior & 57.40 & 9.88 & 20 & 54.85 & 6.97 & 13 & & & & 56.21 & 7.96 & 19 \\
\hline \multicolumn{13}{|l|}{ Parenting Behaviors } \\
\hline APQ-Involvement & 29.24 & 6.97 & 29 & 28.88 & 4.85 & 28 & & & & 30.33 & 6.94 & 25 \\
\hline APQ-Positive Parenting & 25.17 & 3.79 & 30 & 25.54 & 3.48 & 28 & & & & 25.44 & 3.65 & 25 \\
\hline APQ-Poor Monitoring & 10.70 & 2.19 & 29 & 10.81 & 1.98 & 28 & & & & 10.25 & 1.36 & 25 \\
\hline APQ-Inconsistent Discipline & 12.60 & 4.10 & 30 & 13.61 & 5.23 & 28 & & & & 13.76 & 4.31 & 25 \\
\hline APQ-Corporal Punishment & 4.03 & 1.27 & 30 & 4.32 & 1.49 & 28 & & & & 4.36 & 1.82 & 25 \\
\hline \multicolumn{13}{|l|}{ Control Group } \\
\hline \multicolumn{13}{|l|}{ Disruptive Child Behavior } \\
\hline ECBI-Intensity & 103.65 & 34.26 & 36 & 108.16 & 30.02 & 30 & 96.93 & 26.78 & 26 & 101.04 & 24.32 & 25 \\
\hline ECBI-Problem & 8.18 & 7.94 & 36 & 10.12 & 8.56 & 29 & 7.93 & 6.97 & 26 & 8.42 & 6.83 & 25 \\
\hline (C)TRF-Aggressive Behavior & 56.14 & 6.87 & 22 & 57.27 & 6.74 & 11 & & & & 60.33 & 11.57 & 18 \\
\hline \multicolumn{13}{|l|}{ Parenting Behaviors } \\
\hline APQ-Involvement & 29.30 & 5.62 & 36 & 30.03 & 3.93 & 30 & & & & 31.21 & 6.49 & 25 \\
\hline APQ-Positive Parenting & 25.97 & 3.40 & 36 & 26.13 & 3.65 & 31 & & & & 27.28 & 2.51 & 25 \\
\hline APQ-Poor Monitoring & 11.33 & 2.76 & 35 & 11.63 & 4.01 & 30 & & & & 10.48 & 2.04 & 25 \\
\hline APQ-Inconsistent Discipline & 14.47 & 5.30 & 36 & 17.00 & 4.11 & 31 & & & & 15.88 & 3.79 & 25 \\
\hline APQ-Corporal Punishment & 5.00 & 1.94 & 36 & 4.81 & 1.76 & 31 & & & & 4.84 & 2.08 & 25 \\
\hline
\end{tabular}

Note: $\mathrm{ECBI}=$ Eyberg Child Behavior Inventory; $(\mathrm{C}) \mathrm{TRF}=$ Teacher's Report Form or Caregiver-Teacher Report Form; $\mathrm{APQ}=\mathrm{Alabama}$ Parenting Questionnaire.

the random slope for time is significant. The intercept in models is the predicted score on the dependent variable at the first time point. Fixed effect of group indicates whether there is a relation between group status and the mean dependent variable. Positive significant regression coefficients indicate that scores of children in the intervention group are higher in comparison to the control group, whereas negative significant regression coefficients indicate lower scores in the intervention group. Fixed effect of time indicates whether there is a relation between measurement occasions and the dependent variable. Positive significant regression coefficients indicate an increase in the dependent variable across measurement occasions, whereas negative regression coefficients indicate a decrease in the dependent variable.

Disruptive child behavior. Results of final models for disruptive child behavior are presented in Table 2 .
Actual scores and model predicted scores are displayed in Figure 3 and Figure 4.

For intensity of problems (ECBI), results of intentionto-treat analyses revealed a significant Group $\times$ Time interaction $(b=-1.86, p=.04, d=0.30)$, indicating an intervention effect on the intensity of disruptive behavior according to mothers. As shown in Figure 3a, mothers in the intervention group reported a decrease in intensity of disruptive behavior, whereas intensity of disruptive behavior according to mothers remained fairly stable in the control group.

For number of problems (ECBI), a trend toward an interaction effect $(b=-0.34, p=.07, d=0.27)$ was found, indicating a marginal intervention effect on the extent to which child behavior is a problem for the mothers. As shown in Figure 3b, mothers in the intervention group reported a decrease of problems, whereas the extent to which child behavior was 
TABLE 2

Fixed Effects Estimates (Top) and Variance Estimate (Bottom) for Final Models of Change in Disruptive Child Behavior

\begin{tabular}{|c|c|c|c|c|c|c|}
\hline \multirow[b]{2}{*}{ Parameter } & \multicolumn{3}{|c|}{ Intention-to-Treat Analyses } & \multicolumn{3}{|c|}{ Per-Protocol Analyses } \\
\hline & $E C B I-I S$ & $E C B I-P S$ & (C) $T R F$ & $E C B I-I S$ & $E C B I-P S$ & (C) $T R F$ \\
\hline $\begin{array}{l}\text { Fixed Effects } \\
\text { Intercept }\end{array}$ & $102.65^{* * *}(5.48)$ & $7.88^{* * *}(1.36)$ & $55.51^{* * *}(1.46)$ & $102.58^{* * *}(5.54)$ & $7.96^{* * *}(1.39)$ & $55.64^{* * *}(1.49)$ \\
\hline Group & $3.19 \quad(6.39)$ & $2.53 \quad(1.66)$ & $3.52^{\dagger} \quad(1.85)$ & $7.78 \quad(6.81)$ & $4.72^{*} \quad(1.77)$ & $3.88^{\dagger} \quad(2.01)$ \\
\hline Time & $-0.15 \quad(0.89)$ & $0.02 \quad(0.17)$ & $(0.45)$ & $(0.86)$ & $(0.17)$ & $(0.45)$ \\
\hline $\begin{array}{l}\text { Group } \times \text { Time } \\
\text { Random Parameter }\end{array}$ & $-1.86^{*} \quad(1.03)$ & $-0.34^{\dagger} \quad(0.22)$ & $-0.75^{\dagger} \quad(0.47)$ & $-2.67^{* *}(1.05)$ & $-0.55^{*} \quad(0.25)$ & $-0.77^{\dagger} \quad(0.48)$ \\
\hline Slope & $8.22^{* * *}(2.87)$ & $0.56^{* * *}(0.75)$ & $0.34^{*} \quad(0.58)$ & $7.14 * * *(2.67)$ & $0.49^{* * *}(0.70)$ & $(0.53)$ \\
\hline ICC Level 2 & 0.48 & 0.25 & 0.62 & 0.45 & 0.31 & 0.58 \\
\hline ICC Level 3 & 0.31 & 0.54 & 0.15 & 0.34 & 0.45 & 0.10 \\
\hline
\end{tabular}

Note: B values, with standard errors in parentheses. ECBI-IS = Eyberg Child Behavior Inventory-Intensity scale; ECBI-PS = Eyberg Child Behavior Inventory-Problem scale; $(C)$ TRF $=$ Teacher's Report Form or Caregiver-Teacher Report Form-Aggressive behavior; ICC $=$ intraclass correlation; Time $=$ assessment wave.

${ }^{\dagger} p<.10 .{ }^{*} p<.05 .{ }^{* *} p<.01 .{ }^{* * *} p<.001$ (one-tailed for Group $\times$ Time; two-tailed for other parameters).

\section{ECBI-intensity}

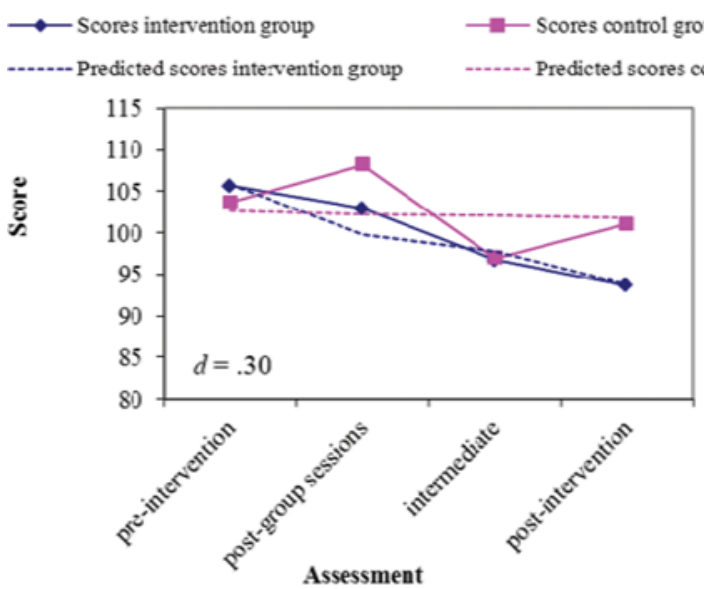

(a)

(C)TRF-aggressive behavior

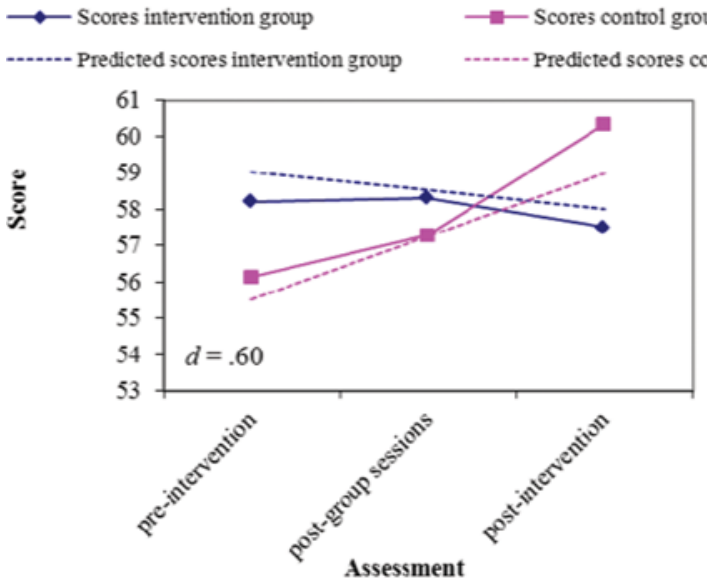

(c)
ECBI-problem
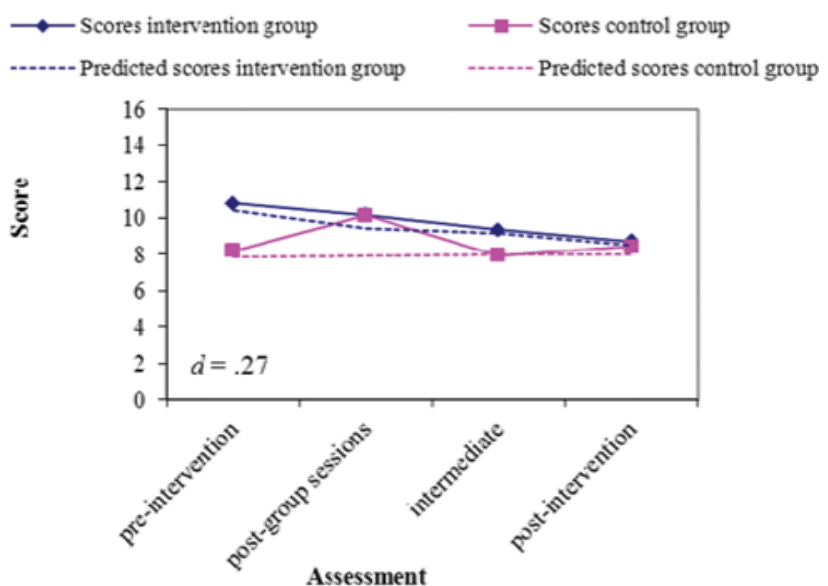

(b)

APQ-inconsistent discipline

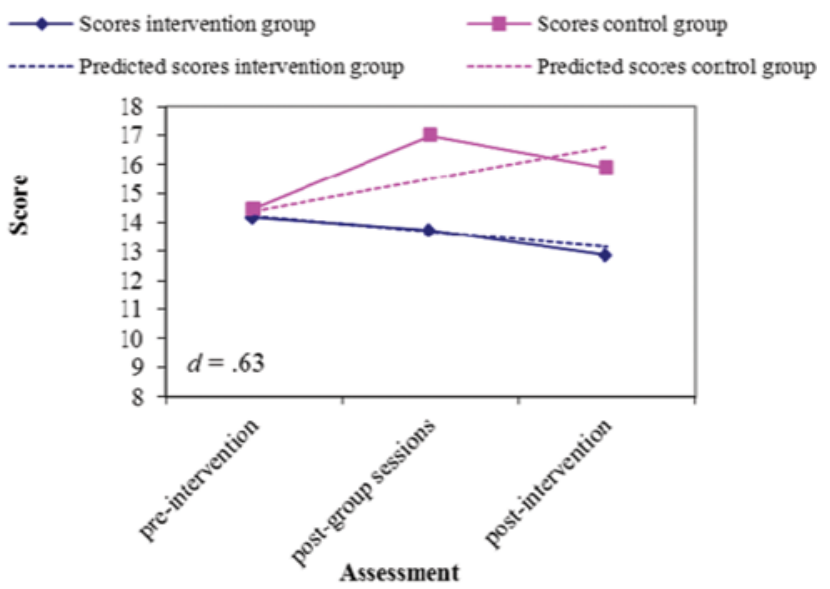

(d)

FIGURE 3 Effect of group on intensity, problem, aggressive behavior, and inconsistent discipline in intention-to-treat analyses. Note. ECBI = Eyberg Child Behavior Inventory; $(\mathrm{C}) \mathrm{TRF}=$ Caregiver-Teacher Report Form; APQ = Alabama Parenting Questionnaire. (Figure appears in color online.) 
ECBI-intensity

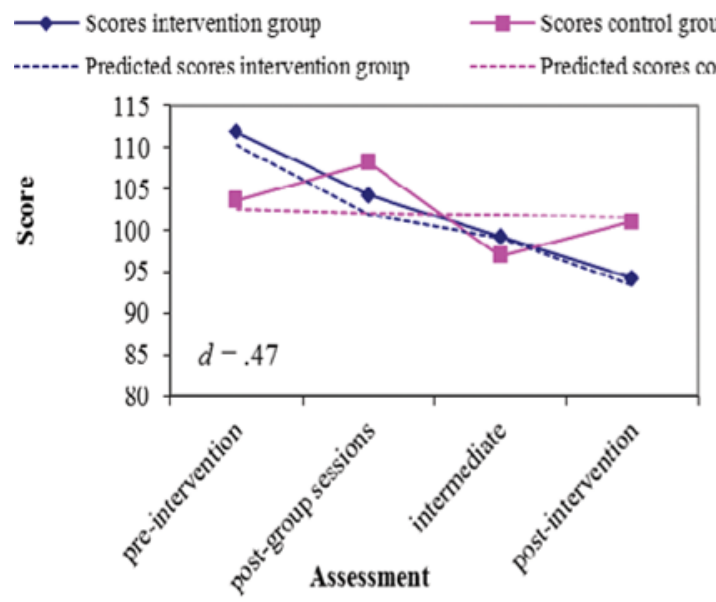

(a)

(C)TRF-aggressive behavior

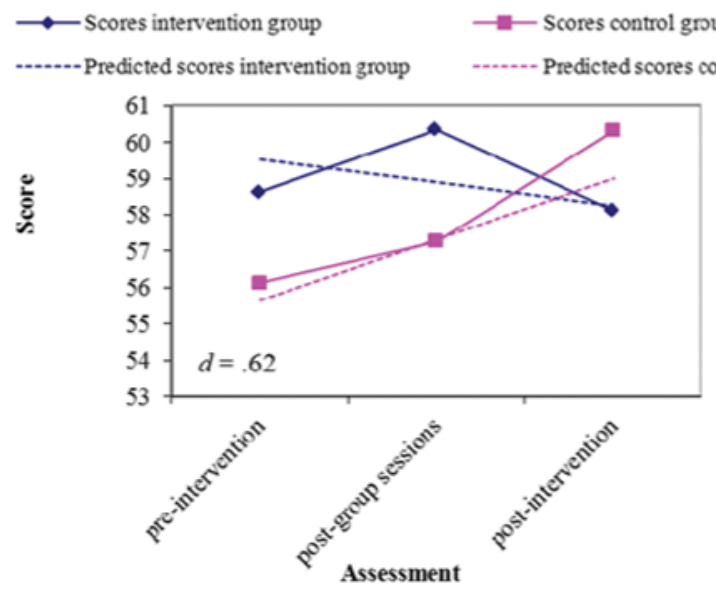

(c)
ECBI-problem

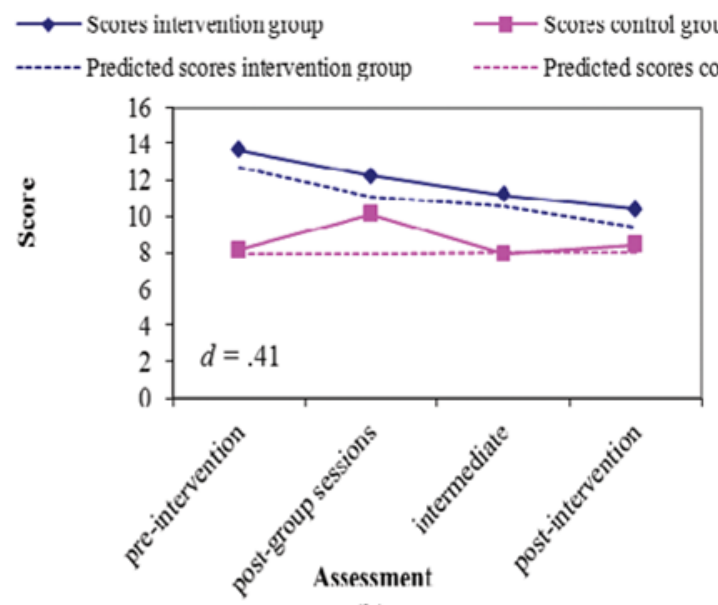

(b)

APQ-inconsistent discipline

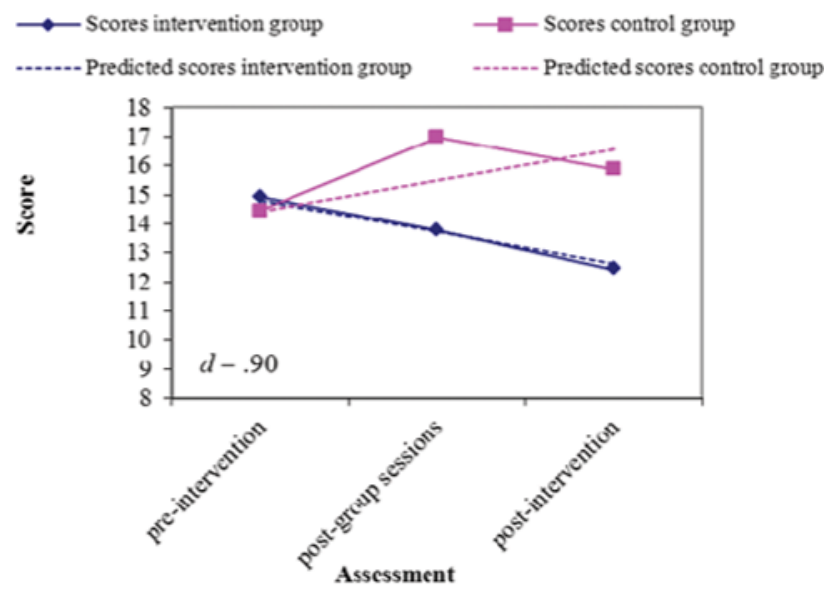

(d)

FIGURE 4 Effect of group on intensity, problem, aggressive behavior and inconsistent discipline in per-protocol analyses. Note. ECBI $=$ Eyberg Child Behavior Inventory; (C)TRF = Caregiver-Teacher Report Form; APQ = Alabama Parenting Questionnaire. (Figure appears in color online.)

considered a problem remained fairly stable in the control group.

For aggressive behavior ((C)TRF) intention-to-treat analyses revealed a trend toward a significant Group $\times$ Time interaction $(b=-0.75, p=.06, d=0.60)$, indicating a marginal intervention effect on disruptive behavior according to teachers and childcare staff, who were blind to allocation. As shown in Figure 3c, teachers and childcare staff reported that children from the intervention group exhibited less aggressive behavior, whereas an increase of aggressive behavior was reported for children from the control group.

In per-protocol analyses, a similar picture was seen, except for number of problems (ECBI). An intervention effect was found for intensity of disruptive behavior according to mothers $(b=-2.67, p=.01, d=0.47)$. Intervention explained $9.2 \%$ of slope variance, or the differences in the regression coefficients over time between families. As shown in Figure $4 \mathrm{a}$, mothers in the intervention group reported a decrease in intensity of disruptive behavior, whereas intensity of disruptive behavior according to mothers remained fairly stable in the control group. For the number of problems according to mothers, per-protocol analyses revealed a significant Group $\times$ Time interaction $(b=-0.55$, $p=.02, d=0.41)$. Intervention explained $9.2 \%$ of slope variance. As shown in Figure 4b, although more problems were reported in the intervention group than in the control group, mothers in the intervention group reported a decrease in problems, whereas the extent to which child behavior was considered a problem remained fairly stable in the control group. A trend toward an interaction effect was found for disruptive behavior according to teachers and childcare staff 


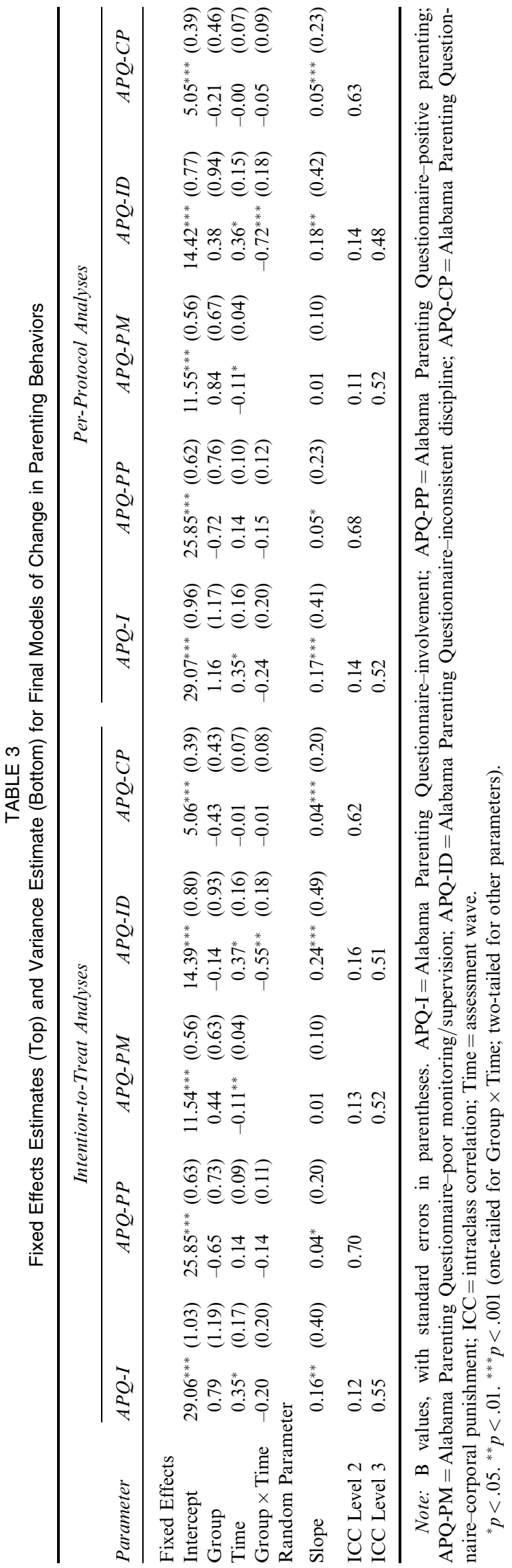

$(b=-0.77, p=.05, d=0.62)$. Intervention explained $24.2 \%$ of slope variance. As shown in Figure 4c, teachers and childcare staff reported that children from the intervention group exhibited less aggressive behavior, whereas an increase of aggressive behavior was reported for children from the control group.

Parenting behaviors. Results of final models for parenting behaviors are presented in Table 3. Actual model predicted scores are displayed in Figure 3 and Figure 4.

For inconsistent discipline, results of intention-totreat analyses revealed a significant Group $\times$ Time interaction $(b=-0.55, p=.002, d=0.63)$, indicating an intervention effect on inconsistency of discipline according to mothers. As shown in Figure 3d, mothers in the intervention group reported a decrease in inconsistency of discipline, whereas mothers in the control group reported an increase in inconsistency of discipline.

For involvement, positive parenting and corporal punishment, no significant Group $\times$ Time interactions were found, indicating no differences in trends between the intervention group and control group. Mothers reported, on average, increasing involvement $(b=0.35$, $p=.04$ ). For poor monitoring, no slope variance regarding assessment waves was found. That is, there were no differences in change in poor monitoring across families. On average, less poor monitoring was reported over time $(b=-0.11, p=.01)$.

A similar picture was seen in per-protocol analyses. An intervention effect was found on inconsistent discipline $(b=-0.72, p<.001, d=0.90)$ but not on other parenting behaviors. For inconsistent discipline, intervention explained $31.8 \%$ of slope variance. As shown in Figure 4d, mothers in the intervention group reported a decrease in inconsistency of discipline, whereas mothers in the control group reported an increase in inconsistency of discipline.

\section{DISCUSSION}

Results show short-term effectiveness of parent training for the high-risk, hard-to-reach population of incarcerated mothers and their children. The intervention led to significant benefits on both disruptive child behavior and parenting behavior. Relative to control mothers, intervention mothers reported reductions in their children's intensity of disruptive behavior as well as their own inconsistency of discipline. In addition, a marginal intervention effect on disruptive behavior according to teachers and childcare staff, blind to intervention status, was found. Per-protocol analyses, in which only mothers who attended at least one intervention session were 
included in the intervention group, yielded highly similar results, including an intervention effect on the number of behavior problems reported by mothers.

In line with our hypotheses, there were immediate intervention effects on disruptive child behavior, according to mothers. This finding is consistent with other selective prevention studies, which yielded intervention effects regarding the IYPT (e.g., Brotman et al., 2003; Nilsen, 2007; Webster-Stratton et al., 2001). In a meta-analytic review of the IYPT (Menting et al., in press), a mean effect size of $d=.13$ was found for selective prevention studies, regarding parent-rated child outcomes. Therefore, the results established for disruptive child behavior according to mothers may be considered substantial. Moreover, this study is, to our knowledge, the first parent training study involving incarcerated mothers to show effects on child behavior besides parenting behaviors.

Because the intervention directly targeted parenting, effects on parenting behaviors were also expected. Consistent with our hypotheses, immediate intervention effects on parenting behavior, specifically inconsistent discipline, came with intervention effects on child behavior. Ineffective parenting, including inconsistent discipline, has been linked to delinquent behavior; children of parents who are inconsistent in disciplining show delinquent behavior more often than children of parents more consistent in child discipline (Hoeve et al., 2009). Moreover, inconsistent discipline has been found to mediate maternal distress and child aggression, which is often a precursor to more serious adolescent delinquency (Barry, Dunlap, Lochman, \& Wells, 2009). Therefore, improvements in consistency of discipline seem especially helpful in breaking the intergenerational cycle in this population with high levels of maternal distress (see Menting et al., 2012).

Improvements were found only for maternal report. The intervention yielded only a marginally significant immediate effect on disruptive behavior according to teachers and childcare staff. Differences between parent-rated outcomes and teacher-rated outcomes are common; they may be caused by genuine contextual differences and more similarity in criteria used by different teachers than used by different parents (Scott, 2001), or insufficient generalization of the intervention effect from home to school settings. Perhaps less improvements according to teachers than according to participating mothers were to be expected in this study: Teachers were blind to allocation, whereas intervention mothers might have overreported improvements due to their own efforts and hopes. However, in this study, the teacherrated effect was only just nonsignificant $(p=.054$ in per-protocol analyses), and effect sizes for teacher-rated disruptive behavior were larger than effect sizes for parent-rated disruptive behavior. Probably, we did not have enough statistical power to detect differences between the intervention and control group. Therefore, the marginal intervention effect found, combined with a moderate effect size and blindness to allocation, may be considered promising.

Delivery of a combination of group and individual intervention components in the period around release from incarceration seems fruitful. Effects of IYPT group sessions are noticeable; effectiveness of these sessions is not only reflected in postintervention effects, but appears immediately after group sessions. For example, Figure $3 \mathrm{~d}$ shows decrease in inconsistency of discipline directly after the group sessions. Because improvements continued after these group sessions, addressing parenting skills and contextual challenges during individual home visits may have built on and elaborated processes that started during group sessions.

The benefits of this intervention for incarcerated mothers and their children should be viewed in light of the many challenges families face when mothers resume parenting upon release. Although the intervention primarily targeted parenting and disruptive behavior problems, addressing parenting behaviors is impossible without addressing mother's incarceration and contextual challenges. That is, for instance, feelings of guilt and urgent problems, such as having no income and housing, may predominate to such an extent that the transference of information regarding parenting skills may be otherwise hindered. The intervention accomplished improvements in parenting and disruptive behavior in spite of contextual difficulties and help with contextual difficulties was offered during intervention. However, it is likely that participants still face difficulties, in spite of successfully participating in the intervention. Also, mothers only recently resumed parenting, and the parent-child relation is still in flux after the mother's absence. For parent training, an opportunity to practice parenting behaviors is essential. Therefore, opportunities to practice had to comprise at least role plays during group sessions and real-life practice during weekend leave. Although still not ideal, opportunities to practice were maximized for incarcerated mothers in this study, given the penitentiary regimes and possibilities for mother-child contact. Perhaps changes in sentencing and penitentiary regime may help improve opportunities for mother-child contact. Furthermore, for both incarcerated and formerly incarcerated mothers parenting behaviors at preintervention are relatively new, because mothers are still regaining parenthood and rebuilding the parent-child relationship. The fact that these processes may still be developing might be an advantage for the intervention but also points to possible changes in parenting apart from the intervention. Therefore, a comparison group receiving no intervention is especially necessary in effectiveness 
studies within this population. Last, participation in a parent training and its beneficial effects cannot compensate for the effects of imprisonment on children and the parent-child relationship: Improved parenting is no replacement for missed time.

An obvious limitation is that we could not randomize two of the six recruitment periods in a 2:1 ratio. This pragmatic approach diminished power to detect differences between intervention and control group and might have hampered equality between groups. However, continuation of the intervention was considered more important than control group size, because we promised potential participants a $2: 1$ chance on participation, and some mothers would not be eligible for a new recruitment period given their release from incarceration would then be more than 6 months ago. Furthermore, these two recruitment periods were not systematically different from other waves, as the lower number of participants was due to a smaller number of mothers ending their prison sentences in these periods, rather than, for example, changes in approach or lower consent rates. Moreover, in our analyses we tried to prevent erroneous conclusions due to group differences. First, we examined Group $\times$ Time interaction effects instead of postintervention differences. That is, we examined whether slopes differed between intervention and control group, irrespective of preintervention or postintervention differences. Second, four-level models were run as part of preliminary analyses to examine variance at the group level. Third, we conducted conservative intention-totreat analyses, in which an opt-out group was added to the intervention group. Because the intervention group in intention-to-treat analyses equals the intervention group as randomized, more equality between groups may be assumed in these analyses.

Group sizes in this study are not very large. However, given our nationwide screening and relatively high consent rate, participants are considered almost the entire eligible population. As such, our sample seems to reflect the population of incarcerated mothers caring for children between 2 and 10 years of age in the Netherlands. Moreover, our attrition rate seems to be at least comparable to other intervention studies with female prisoners. Browne (1989), Harm and Thompson (1997), and Sandifer (2008) all reported availability of less than $70 \%$ of their pretest sample during their second assessment. In comparison, $78.4 \%$ of mothers who completed the preintervention assessment were available at postintervention in this study. In addition, although absolute numbers of teacher responses are small, the response rate for approached teachers and childcare staff is considerable (on average $84.6 \%$ ). Furthermore, the relatively small eligible population in the Netherlands demanded the inclusion of children with a relatively large age range. Mothers from the whole country had to travel from their residence to attend the group sessions, and although they were assisted to do this as much as possible (e.g., with tickets, schedules, and reminders), this may have decreased attendance. However, the fact that mothers were willing to travel substantial distances and most mothers attended a substantial number of the group sessions, in spite of transportation difficulties and their own contextual challenges, suggests the feasibility of this intervention for this population. Moreover, nonattendance did not mean that the topic of the session was not covered; mothers were filled in on the session's content as much as possible.

In addition, the use of usual services was not addressed in this study. Although all participants (including control families) were encouraged to use adequate services, there might have been differences between groups in the actual services received. Unfortunately, our data were not suitable to investigate differences in usual services at study entry and/or to control for the use of these services during our intervention. It was not realistic to assess usual services systematically through maternal report during the intake interview: Incarcerated mothers were not able to have contact with usual services themselves for a significant period and may not be well informed regarding contacts by caregivers.

Results immediately after intervention are promising: A hard-to-reach population was reached and short-term intervention effects were found. However, this is just the first step, and effects were not found for all outcome measures. This intervention's ultimate purpose is to break the intergenerational cycle of crime by means of improvements in delinquent mothers' parenting and in their children's behavior; therefore, future research regarding long-term effectiveness is essential in view of this intervention's preventative purpose. Possibly, the "real" preventive character will be more reflected in future results, as so-called sleeper effects may be expected regarding the prevention of disruptive behavior problems and delinquency (Hinshaw, 2002). Therefore, repeated assessments of both parenting and child behavior in the nearby future, as well as examination of arrest and incarceration rates when children reach adulthood, are in process. Because short-term intervention effects were found only for maternal report, a challenging task for future research is the examination of effects according to teachers and official records.

Research should also focus on identification of promotive and protective factors in children affected by maternal incarceration. Identifying children who show relatively few problems or respond relatively well to minor changes in parenting behaviors will help to understand both resiliency in children affected by maternal incarceration and intervention processes. In addition, promotive and protective factors may indicate possibilities for improvements regarding the intervention. 
Addressing contextual challenges likely to disrupt parenting after release seems crucial in helping families affected by maternal incarceration. However, boundaries of what can be accomplished from a parent training are reached relatively soon. That is, mothers' material, personal, and other contextual problems often exceed expertise, and time, of individual team members. Referring mothers to other organizations, including a warm transfer, is possible without formal collaboration. However, clear agreements with organizations will help to guide mothers to skillful parenting, hampered as little as possible by challenges like housing, getting a job, and avoiding destructive relationships.

Last, the effects found in this study are context specific. That is, it is unclear whether the same intervention would yield similar results within other contexts, such as in other countries with other legal systems, other penitentiary regimes, and other social services, or with incarcerated fathers. For example, differences regarding the criminal justice system or contextual challenges after release may affect effectiveness. Therefore, delivery of this specific intervention might need extra efforts to realize sufficient parent-child contact and facilitate group meetings, additional or different help with contextual challenges, and additional adaptations of the intervention. However, whatever the exact context, the vulnerable population of children affected by parental incarceration deserves efforts to deliver and adapt interventions. Although the real preventive nature of the intervention might be more reflected after a significant period and short-term effects were only found for maternal report, the present study demonstrates the immediate effects intervention can have on vulnerable children's lives.

\section{REFERENCES}

Achenbach, T. M., \& Rescorla, L. A. (2000). Manual for ASEBA preschool forms \& profiles. Burlington: Research Center for Children, Youth, and Families, University of Vermont.

Achenbach, T. M., \& Rescorla, L. A. (2001). Manual for the ASEBA school-age forms \& profiles. Burlington: Research Center for Children, Youth, and Families, University of Vermont.

Barlow, J., Kirkpatrick, S., Stewart-Brown, S., \& Davis, H. (2005). Hard-to-reach or out-of-reach? Reasons why women refuse to take part in early interventions. Children \& Society, 19, 199-210. doi:10.1002/CHI.835

Barry, T. D., Dunlap, S. T., Lochman, J. E., \& Wells, K. C. (2009). Inconsistent discipline as a mediator between maternal distress and aggression in boys. Child \& Family Behavior Therapy, 31, 1-19. doi:10.1080/07317100802701186

Bijleveld, C. C. J. H., \& Wijkman, M. (2009). Intergenerational continuity in convictions: A five-generation study. Criminal Behaviour \& Mental Health, 19, 142-155. doi:10.1002/cbm.714

Boudin, K. (1998). Lessons from a mother's program in prison: A psychosocial approach supports women and their children. Women \& Therapy, 21, 103-125. doi:10.1300/J015v21n01_01

Broidy, L. M., Nagin, D. S., Tremblay, R. E., Bates, J. E., Brame, B., Dodge, K. A.,... Vitaro, F. (2003). Developmental trajectories of childhood disruptive behavior disorders and adolescent delinquency: A six-site, cross-national study. Developmental Psychology, 39, 222-245. doi:10.1037/0012-1649.39.2.222

Brotman, L. M., Gouley, K. K., Chesir-Teran, D., Dennis, T., Klein, R. G., \& Shrout, P. (2005). Prevention for preschoolers at high risk for conduct problems: Immediate outcomes on parenting practices and child social competence. Journal of Clinical Child \& Adolescent Psychology, 34, 724-734. doi:10.1207/s15374424jccp3404_14

Brotman, L. M., Klein, R. G., Kamboukos, D., Brown, E. J., Coard, S. I., \& Sosinsky, L. S. (2003). Preventive intervention for urban, low-income preschoolers at familial risk for conduct problems: A randomized pilot study. Journal of Clinical Child and Adolescent Psychology, 32(2), 246-257. doi:10.1207/S1537 4424JCCP3202_10

Browne, D. H. (1989). Incarcerated mothers and parenting. Journal of Family Violence, 4(2), 211-221. doi:10.1007/BF01006630

Bullard, L., Wachlarowicz, M., DeLeeuw, J., Snyder, J., Low, S., Forgatch, M., \& DeGarmo, D. (2010). Effects of the Oregon model of Parent Management Training (PMTO) on marital adjustment in new stepfamilies: A randomized trial. Journal of Family Psychology, 24, 485-496. doi:10.1037/a0020267

Cassidy, J., Ziv, Y., Stupica, B., Sherman, L. J., Butler, H., Karfgin, A.,... Powell, B. (2010). Enhancing attachment security in the infants of women in a jail-diversion program. Attachment \& Human Development, 12, 333-353. doi:10.1080/14616730903416955

Conger, R. D., Neppl, T., Kim, K. J., \& Scaramella, L. (2003). Angry and aggressive behavior across three generations: A prospective, longitudinal study of parents and children. Journal of Abnormal Child Psychology, 31, 143-160. doi:10.1023/A:1022570107457

Dallaire, D. H. (2007). Children with incarcerated mothers: Developmental outcomes, special challenges and recommendations. Journal of Applied Developmental Psychology, 28, 15-24. doi:10.1016/j. appdev.2006.10.003

Dodge, K. A., Coie, J. D., \& Lynam, D. (2006). Aggression and antisocial behavior in youth. In N. Eisenberg (Ed.), Handbook of child psychology: Vol. 3 social, emotional, and personality development (6th ed., pp. 719-788). New York, NY: Wiley.

Eddy, B. A., Powell, M. J., Szubka, M. H., McCool, M. L., \& Kuntz, S. (2001). Challenges in research with incarcerated parents and importance in violence prevention. American Journal of Preventive Medicine, 20(1, Suppl. 1), 56-62. doi:10.1016/S0749-3797(00)00273-7

Eddy, J. M., Kjellstrand, J., Martinez, C. R., \& Newton, R. (2010). Theory-based multimodal parenting intervention for incarcerated parents and their children. In J. M. Eddy \& J. Poehlmann (Eds.), Children of incarcerated parents: $A$ handbook for researchers and practitioners (pp. 237-261). Washington, DC: The Urban Institute Press.

Eddy, J. M., Martinez, C. R., Schiffmann, T., Newton, R., Olin, L., Leve, L., ... Shortt, J. W. (2008). Development of a multisystemic parent management training intervention for incarcerated parents, their children and families. Clinical Psychologist, 12, 86-98. doi:10.1080/13284200802495461

Eyberg, S. M., \& Pincus, D. (1999). Eyberg Child Behavior Inventory \& Sutter-Eyberg Student Behavior Inventory-Revised. Odessa, FL: Psychological Assessment Resources.

Goldstein, R. B., Dawson, D. A., Smith, S. M., \& Grant, B. F. (2012). Antisocial behavioral syndromes and 3-year quality-of-life outcomes in United States adults. Acta Psychiatrica Scandinavica, 126, 137-150. doi:10.1111/j.1600-0447.2012.01848.x

Gryczkowski, M. R., Jordan, S. S., \& Mercer, S. H. (2010). Differential relations between mothers' and fathers' parenting practices and child externalizing behavior. Journal of Child and Family Studies, 19, 539-546. doi:10.1007/s10826-009-9326-2

Harm, N. J., \& Thompson, P. J. (1997). Evaluating the effectiveness of parent education for incarcerated mothers. Journal of Offender Rehabilitation, 24, 135-152. doi:10.1300/J076v24n03_08 
Hinshaw, S. P. (2002). Prevention/intervention trials and developmental theory: Commentary on the Fast Track special section. Journal of Abnormal Child Psychology, 30, 53-59. doi:10.1023/A:101427 9015195

Hoeve, M., Dubas, J. S., Eichelsheim, V. I., Van der Laan, P. H., Smeenk, W., \& Gerris, J. R. M. (2009). The relationship between parenting and delinquency: A meta-analysis. Journal of Abnormal Child Psychology, 37, 749-775. doi:10.1007/s10802-009-9310-8

Hughes, J. R., \& Gottlieb, L. N. (2004). The effects of the Webster-Stratton parenting program on maltreating families: Fostering strengths. Child Abuse \& Neglect, 28, 1081-1097. doi:10.1016/j.chiabu.2004.02.004

Jouriles, E., McDonald, R., Rosenfield, D., Stephens, N., Corbitt-Shindler, D., \& Miller, P. C. (2009). Reducing conduct problems among children exposed to intimate partner violence: A randomized clinical trial examining effects of project support. Journal of Consulting and Clinical Psychology, 77, 705-717. doi:10.1037/a0015994

Keij, I. (2000). Standaard definitie allochtonen [Standard definition of foreigners]. Index, 10, 24-25.

Lipsey, M. W., \& Derzon, J. H. (1998). Predictors of violent or serious delinquency in adolescence and early adulthood. In R. Loeber \& D. P. Farrington (Eds.), Serious \& violent juvenile offenders: Risk factors and successful interventions (pp. 86-105). Thousand Oaks, CA: Sage.

Loeber, R., \& Keenan, K. (1994). Interaction between conduct disorder and its comorbid conditions: Effect of age and gender. Clinical Psychology Review, 14, 497-523. doi:10.1016/0272-7358(94)90015-9

Loper, A. B., \& Novero, C. M. (2010). Parenting programs for prisoners. In J. M. Eddy \& J. Poehlmann (Eds.), Children of incarcerated parents: A handbook for researchers and practitioners (pp. 189-215). Washington, DC: The Urban Institute Press.

Loper, A. B., \& Tuerk, E. H. (2011). Improving the emotional adjustment and communication patters of incarcerated mothers: Effectiveness of a prison parenting intervention. Journal of Child and Family Studies, 20, 89-101. doi:10.1007/s10826-010-9381-8.

McCart, M. R., Priester, P. E., Davies, W. H., \& Azen, R. (2006). Differential effectiveness of behavioral parent-training and cognitive-behavioral therapy for antisocial youth: A meta-analysis. Journal of Abnormal Child Psychology, 34, 527-543. doi:10.1007/ s10802-006-9031-1

Menting, A. T. A., De Castro, Bram O., \& Matthys, W. (in press). Effectiveness of the Incredible Years parent training to modify disruptive and prosocial child behavior: A meta-analytic review. Clinical Psychology Review.

Menting, A. T. A., De Castro, Bram O., \& Matthys, W. (2012). Risky motherhood: Parenting, cognitive distortions, and distress in mothers being released from incarceration. Manuscript submitted for publication.

Moffitt, T. E. (2005). The new look of behavioral genetics in developmental psychopathology: Gene-Environment interplay in antisocial behaviors. Psychological Bulletin, 131, 533-554. doi:10.1037/ 0033-2909.131.4.533

Moore, A., \& Clement, M. (1998). Effects of parenting training for incarcerated mothers. Journal of Offender Rehabilitation, 27, 57-72. doi:10.1300/J076v27n01_05

Murray, J., Farrington, D. P., \& Sekol, I. (2012). Children's antisocial behavior, mental health, drug use, and educational performance after parental incarceration: A systematic review and meta-analysis. Psychological Bulletin, 138, 175-210. doi:10.1037/a0026407

Nilsen, W. (2007). Fostering futures: A preventive intervention program for school-age children in foster care. Clinical Child Psychology and Psychiatry, 12, 45-63. doi:10.1177/1359104507071055
Olds, D. L., Robinson, J., O’Brien, R., Luckey, D. W., Pettitt, L. M., Henderson, C. R., ... Talmi, A. (2002). Home visiting by paraprofessionals and by nurses: A randomized, controlled trial. Pediatrics, 110, 486-496.

Palusci, V. J., Crum, P., Bliss, R., \& Bavolek, S. J. (2008). Changes in parenting attitudes and knowledge among inmates and other at-risk populations after a family nurturing program. Children and Youth Services Review, 30, 79-89. doi:10.1016/j.childyouth.2007.06.006

Raudenbush, S. W., Bryk, A. S., \& Congdon, R. T. (2004). HLM: Hierarchical linear and nonlinear modeling for Windows (version 6.08). Lincolnwood, IL: Scientific Software International.

Reid, J. B., Patterson, G. R., \& Snyder, J. (2002). Antisocial behavior in children and adolescents: A developmental analysis and model of intervention. Washington, DC: American Psychological Association.

Rhule, D. M., McMahon, R. J., \& Spieker, S. J. (2004). Relation of adolescent mothers' history of antisocial behavior to child conduct problems and social competence. Journal of Clinical Child \& Adolescent Psychology, 33, 524-535. doi:10.1207/s15374424jccp3303_10

Sameroff, A. J., \& Seifer, R. (1993). Stability of intelligence from preschool to adolescence: The influence of social and family risk factors. Child Development, 64, 80-97. doi:10.1111/1467-8624.ep9309015108

Sandifer, J. L. (2008). Evaluating the efficacy of a parenting program for incarcerated mothers. Prison Journal, 88, 423-445. doi:10.1177/ 0032885508322533

Scott, S. (2001). Deciding whether interventions for antisocial behaviour work: Principles of outcome assessment, and practice in a multicentre trial. European Child \& Adolescent Psychiatry, 10(Suppl. 1), 59-70. doi:10.1007/s007870170007

Shaw, D. S., Lacourse, E., \& Nagin, D. S. (2005). Developmental trajectories of conduct problems and hyperactivity from ages 2 to 10. Journal of Child Psychology and Psychiatry, 46, 931-942. doi:10.1111/j.1469-7610.2004.00390.x

Shelton, K. K., Frick, P. J., \& Wootton, J. (1996). Assessment of parenting practices in families of elementary school-age children. Journal of Clinical Child Psychology, 25, 317-329. doi:10.1207/ s15374424jccp2503_8

Simons, R. L., Stewart, E., Gordon, L. C., Conger, R. D., \& Elder, G. H., Jr. (2002). A test of life-course explanations for stability and change in antisocial behavior from adolescence to young adulthood. Criminology, 40, 401-434. doi:10.1111/j.1745-9125.2002.tb00961.x

Thompson, P. J., \& Harm, N. J. (2000). Parenting from prison: Helping children and mothers. Issues in Comprehensive Pediatric Nursing, 23, 61-81. doi:10.1080/01460860050121402

Thornberry, T. P., Freeman-Gallant, A., Lizotte, A. J., Krohn, M. D., \& Smith, C. A. (2003). Linked lives: The intergenerational transmission of antisocial behavior. Journal of Abnormal Child Psychology, 31, 171-184. doi:10.1023/A:1022574208366

Webster-Stratton, C. (1992). The Incredible Years: A trouble-shooting guide for parents of children ages 3-8 years. Toronto, Canada: Umbrella Press.

Webster-Stratton, C. (2001). The Incredible Years: Parents and children videotape series: A parenting course (BASIC). Seattle, WA: Incredible Years.

Webster-Stratton, C. (2002). The Incredible Years: Parents and children videotape series: A parenting course (ADVANCE). Seattle, WA: Incredible Years.

Webster-Stratton, C. (2009). Affirming diversity: Multi-cultural collaboration to deliver the Incredible Years parent programs. International Journal of Child Health and Human Development, 2, 17-32.

Webster-Stratton, C., Reid, M. J., \& Hammond, M. (2001). Preventing conduct problems, promoting social competence: A parent and teacher training partnership in Head Start. Journal of Clinical Child Psychology, 30, 283-302. doi:10.1207/S15374424JCCP3003_2 